\title{
Isquemia e reperfusão de músculo sóleo de ratos sob ação da pentoxifilina
}

\author{
Ischemia and reperfusion of the soleus muscle of rats \\ with pentoxifylline

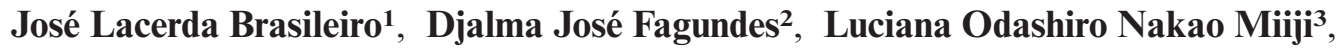
Celina Tizuko Fujiama Oshima ${ }^{4}$, Roberto Teruya ${ }^{5}$, Guido Marks ${ }^{5}$, Celso Massaschi Inouye ${ }^{5}$, Maldonat Azambuja Santos ${ }^{6}$
\end{abstract}

\section{Resumo}

Contexto: A reperfusão de músculo esquelético piora as lesões já presentes no período de isquemia, pois a produção de espécies reativas de oxigênio, associadas à intensa participação de neutrófilos, amplia a reação inflamatória que induz alterações teciduais.

Objetivo: Avaliar as alterações morfológicas e imuno-histoquímicas de músculo esquelético (sóleo) de ratos submetidos a isquemia e reperfusão com pentoxifilina.

Métodos: Sessenta ratos foram submetidos a isquemia do membro pélvico, por 6 horas, pelo clampeamento da artéria ilíaca comum esquerda. Após isquemia, os animais do grupo A $(n=30)$ foram observados por 4 horas, e os do grupo $B(n=30)$, por 24 horas. Seis animais constituíram o grupo simulado. Administrou-se pentoxifilina apenas no período de reperfusão em A2 $(n=10)$ e B2 $(n=10)$ e nos períodos de isquemia e reperfusão em A3 $(n=$ 10) e B3 $(\mathrm{n}=10)$. O músculo sóleo foi avaliado por análise histológica (dissociação de fibras, infiltrado leucocitário, necrose) e imuno-histoquímica (apoptose pela expressão da caspase-3). Foram aplicados os testes não-paramétricos de Kruskal-Wallis e Mann-Whitney $(\mathrm{p}<0,05)$.

Resultados: As alterações foram mais intensas no grupo B1, com médias de escore da dissociação de fibras musculares de $2,16 \pm 0,14$, infiltrado neutrofílico de $2,05 \pm 0,10$ e expressão da caspase- 3 na área perivascular de 4,30 $\pm 0,79$; e menos intensas no grupo $\mathrm{A} 3$, com respectivas médias de $0,76 \pm 0,16$, $0,92 \pm 0,10$ e $0,67 \pm 0,15(\mathrm{p}<0,05)$. A caspase- 3 mostrou-se mais expressiva no grupo B1 na área perivascular, com média de 4,30 \pm 0,79, em comparação com o grupo B1 na área perinuclear, com média de $0,91 \pm 0,32(\mathrm{p}<0,05)$.

Conclusões: As lesões são mais intensas quando o tempo de observação é maior após a reperfusão, e a pentoxifilina atenua essas lesões, sobretudo quando usada no início das fases de isquemia e de reperfusão.

Palavras-chave: Isquemia, reperfusão, músculo esquelético, ratos, pentoxifilina, caspases, apoptose.

\begin{abstract}
Background: Reperfusion of the skeletal muscle worsens existing lesions during ischemia, since the production of reactive oxygen species, associated with intense participation of neutrophils, increases the inflammatory reaction that induces tissue changes.

Objective: To evaluate the morphological and immunohistochemical changes of the skeletal (soleus) muscle of rats submitted to ischemia and reperfusion with pentoxifylline.

Methods: Sixty rats were submitted to ischemia of the pelvic limb for 6 hours induced by clamping the left common iliac artery. After ischemia, group A animals $(n=30)$ were observed for 4 hours and group B animals $(n=30)$ for 24 hours. Six animals constituted the sham group. Pentoxifylline was applied only in the reperfusion period $\mathrm{A} 2(\mathrm{n}=10)$ and $\mathrm{B} 2(\mathrm{n}=10)$, and in ischemia and reperfusion periods in $A 3(n=10)$ and $B 3(n=10)$. The soleus muscle was evaluated by histological (fiber disruption, leukocyte infiltrate, necrosis) and immunohistochemical (apoptosis through caspase-3 expression) analysis. The non-parametric tests Kruskal-Wallis and Mann-Whitney $(\mathrm{p}<0.05)$ were applied.
\end{abstract}

Results: The changes were more intense in group B1, with fiber disruption mean scores of $2.16 \pm 0.14$; neutrophilic infiltrate of $2.05 \pm 0.10$; and caspase- 3 expression in the perivascular area of $4.30 \pm 0.79$; and less intense in group A3, with means of $0.76 \pm 0.16 ; 0.92 \pm 0.10 ; 0.67 \pm 0,15$, respectively $(\mathrm{p}<0.05)$. Caspase- 3 was more expressive in group B1 in the perivascular area, with mean of $4.30 \pm 0.79$ when compared with group $\mathrm{B} 1$ in the perinuclear area, with mean of $0.91 \pm 0.32(\mathrm{p} \leq 0.05)$.

Conclusions: The lesions were more intense when observation time was longer after reperfusion, and pentoxifylline attenuated these lesions, above all when used in the beginning of ischemia and reperfusion phases.

Keywords: Ischemia, reperfusion, skeletal muscle, rats, pentoxifylline, caspases, apoptosis.

1. Membro titular, SBACV. Preceptor, Residência Médica em Cirurgia Vascular, Hospital Universitário, Universidade Federal de Mato Grosso do Sul (UFMS), Campo Grande, MS.

2. Professor adjunto, Disciplina de Técnica Operatória e Cirurgia Experimental, Universidade Federal de São Paulo- Escola Paulista de Medicina (UNIFESP-EPM), São Paulo, SP.

3. Mestre. Professora de Patologia, UFMS, Campo Grande, MS.

4. Professora, Laboratório de Patologia Molecular, Departamento de Patologia, UNIFESP-EPM, São Paulo, SP.

5. Professores, Departamento de Clínica Cirúrgica, UFMS, Campo Grande, MS.

6. Coordenador, Comissão de Residência Médica (COREME), Hospital Universitário, UFMS, Campo Grande, MS. Supervisor, Residência Médica em Cirurgia Vascular, Hospital Universitário, UFMS, Campo Grande, MS.

Pesquisa desenvolvida no Programa de Pós-Graduação em Cirurgia e Experimentação, UNIFESP-EPM, São Paulo, SP.

Artigo submetido em 30.11.06, aceito em 27.02.07.

J Vasc Bras 2007;6(1):50-63.

Copyright $\odot 2007$ by Sociedade Brasileira de Angiologia e de Cirurgia Vascular 


\section{Introdução}

A reperfusão de músculo esquelético, embora necessária para reverter o estado isquêmico, piora as lesões já presentes no período de isquemia, pois, com a chegada do oxigênio e a produção de espécies reativas de oxigênio associadas à intensa participação de neutrófilos, amplia a reação inflamatória, com conseqüentes alterações morfológicas, como edema intersticial e celular, infiltrado leucocitário, trombose da microcirculação, apoptose ou necrose tecidual ${ }^{1-6}$.

O músculo sóleo tem sido utilizado para estudo das alterações oxidativas por predominarem, em sua constituição, as fibras tipo I (91\%), sendo considerado um modelo sensível ao estresse oxidativo ${ }^{4,7}$. Contudo, para provocar a isquemia do músculo sóleo, é preciso considerar que a vascularização do membro pélvico do rato apresenta uma exuberante presença de circulação colateral no território da artéria ilíaca externa e artéria femoral comum; assim, esses aspectos devem ser considerados na escolha do modelo a ser utilizado para provocar a isquemia ${ }^{7,8}$. No modelo de isquemia induzido pelo clampeamento arterial isolado, evita-se a compressão direta do leito venoso, assim como o trauma muscular direto provocado pelo modelo que utiliza o torniquete, permitindo, dessa forma, a preservação das respostas vasomotora e venosa durante a reperfusão ${ }^{2,8}$.

A pentoxifilina tem se mostrado promissora. É uma droga hemorreológica padrão, para uso em doenças vasculares crônicas, e vem sendo testada para modular as alterações morfológicas e bioquímicas provocadas pela isquemia e reperfusão em vários órgãos e tecidos, como: coração ${ }^{9}$, ovário ${ }^{10}$, músculo ${ }^{11,12}$, fígado ${ }^{13}$, pulmão ${ }^{14}$, medula espinhal ${ }^{15}$ e intestino ${ }^{16}$. Ela atua como inibidor da fosfodiesterase, que induz aumento de adenosina monofosfato cíclico, melhorando o fluxo da microcirculação; diminui a migração de neutrófilos; reduz a liberação de citocinas; aumenta a produção de prostaciclinas; e reduz a liberação de espécies reativas de oxigênio. Essas propriedades têm sido responsabilizadas pela sua ação de atenuação sobre as lesões de isquemia e reperfusão ${ }^{6,9,16,17}$.
O objetivo deste estudo é criar um modelo de isquemia e reperfusão em ratos para avaliar as alterações morfológicas e imuno-histoquímicas do músculo sóleo sob a ação da pentoxifilina.

\section{Método}

O projeto de pesquisa foi aprovado pelo Comitê de Ética em Pesquisa da Universidade Federal de Mato Grosso do Sul (UFMS) e da Escola Paulista de Medicina da Universidade Federal de São Paulo (UNIFESPEPM), conforme os protocolos de pesquisa com os respectivos números: 38/2003 e 0334/04. Todos os animais foram manipulados de acordo com os princípios éticos na experimentação animal da União Internacional Protetora dos Animais e da Lei Federal n ${ }^{\circ}$ 663, de 8 de maio de 1979.

Foram utilizados 66 ratos (Rattus norvegicus albinus) da linhagem Wistar EPM-1, machos, adultos, com peso médio de 335,6 g, provenientes do biotério da UFMS, onde foram realizados os procedimentos operatórios.

Aleatoriamente, os animais foram distribuídos em grupo simulado (GS), grupo A e grupo B, segundo os tipos de procedimento experimental:

- GS $(n=6)$ : animais que foram submetidos a anestesia, laparotomia, sem isquemia de membro posterior e coleta de músculo sóleo após 4 horas $(n=3)$ e 24 horas $(n=3)$ de observação;

- Grupo A ( $\mathrm{n}=30)$ : animais que foram submetidos a anestesia, laparotomia, isquemia de 6 horas do membro posterior e coleta de músculo sóleo após 4 horas de reperfusão;

- Grupo B ( $=30)$ : animais que foram submetidos a anestesia, laparotomia, isquemia de 6 horas do membro posterior e coleta de músculo sóleo após 24 horas de reperfusão.

Os animais dos grupos A e $\mathrm{B}$ foram redistribuídos em subgrupos 1 (A1 e B1), 2 (A2 e B2) e 3 (A3 e B3) de acordo com a aplicação da pentoxifilina:

- A1 e B1 (n = 10): animais que não receberam a droga, quer no período de isquemia, quer no período de reperfusão; 
- A2 e B2 $(n=10)$ : animais que receberam a droga somente no momento imediatamente anterior ao início da reperfusão (3 minutos);

- A3 e B3 $(\mathrm{n}=10)$ : animais que receberam a droga tanto no momento imediatamente anterior ao início da isquemia (3 minutos) quanto no momento imediatamente anterior ao início da reperfusão (3 minutos).

Os animais foram anestesiados com injeção intraperitoneal de solução de 2:1 de cloridrato de quetamina $\left(50 \mathrm{mg} \cdot \mathrm{mL}^{-1}\right)$ e xilazina $\left(20 \mathrm{mg} \cdot \mathrm{mL}^{-1}\right)$, respectivamente, na dose de $0,1 \mathrm{~mL} \cdot 100 \mathrm{~g}^{-1}$. Nalbufina $\left(\right.$ Nubain $\left.^{\circledR}\right)$ foi administrada na dose de $2 \mathrm{mg} \cdot \mathrm{kg}^{-1}$, por via subcutânea, para analgesia durante o tempo que os animais ficaram em observação, nos períodos de isquemia ou de reperfusão. A pentoxifilina $\left(\operatorname{Trental}^{\circledR}\right)$, na dose de $40 \mathrm{mg} \cdot \mathrm{kg}^{-1}$, foi aplicada por via intraperitoneal, em um volume de 2 mL. Fez-se a laparotomia mediana para colocação de miniclampe vascular, por um período de 6 horas, em artéria ilíaca comum esquerda (Figura 1).

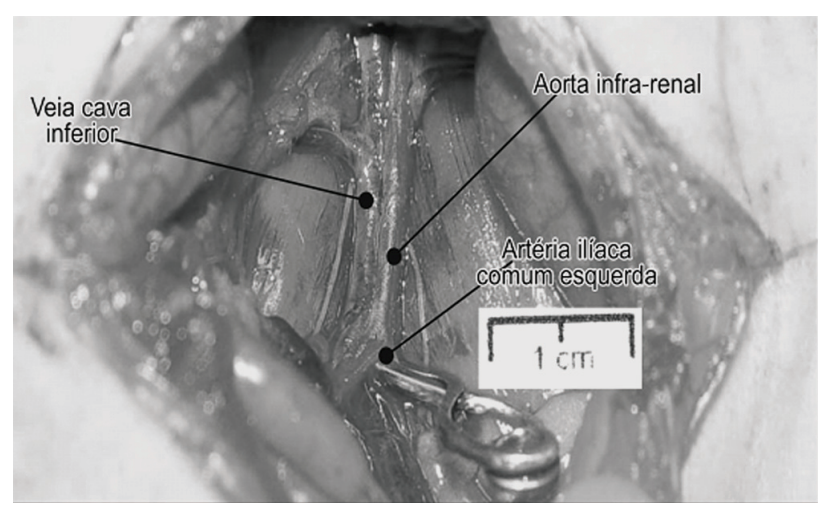

Figura 1 - Fotografia mostrando o isolamento e clampeamento da artéria ilíaca comum esquerda do rato

O miniclampe vascular foi retirado após 6 horas de isquemia, para observação de acordo com os grupos de 4 e 24 horas de reperfusão com água e dieta à vontade.

Após o período de reperfusão desejado, foi realizada a eutanásia por exsangüinação total do animal, com morte por hipovolemia.

O músculo foi dissecado e excisado (Figura 2) para estudo morfológico e imuno-histoquímico. A peça foi submersa em formol tamponado (para cada litro de formol a $10 \%$, adicionou-se $4 \mathrm{~g}$ de fosfato de sódio monobásico anidro e $6,5 \mathrm{~g}$ de fosfato de sódio dibásico anidro) para avaliação morfológica (hematoxilinaeosina) e imuno-histoquímica (expressão da caspase-3). $\mathrm{O}$ processamento e a análise da microscopia óptica foram realizados pelo patologista do Departamento de Patologia da UNIFESP-EPM, sem o prévio conhecimento dos grupos experimentais. O processamento e a quantificação imuno-histoquímica foram realizados no Laboratório de Patologia Molecular do Departamento de Patologia.

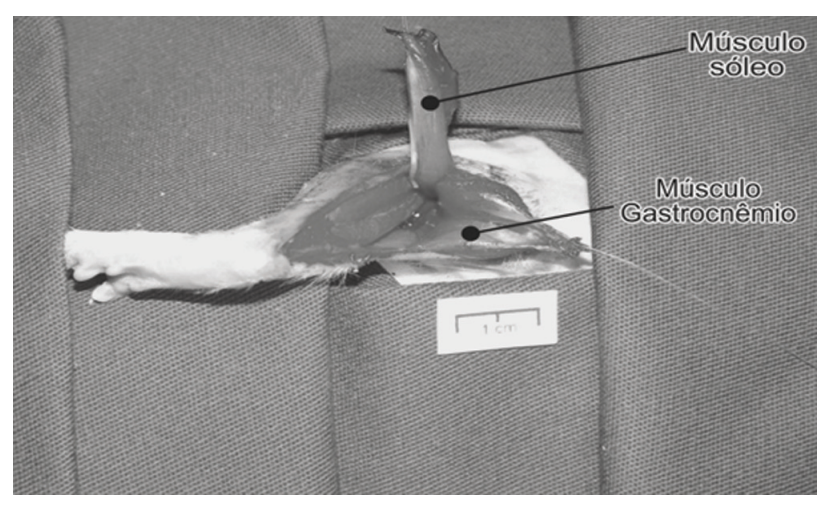

Figura 2 - Foto da dissecção da face posterior da perna do rato, identificando-se os músculos gastrocnêmio e sóleo

Na análise por microscopia óptica, em magnificação de 100 e 400 vezes, as lâminas coradas por hematoxilinaeosina foram observadas em relação aos seguintes aspectos da morfologia das células musculares: dissociação de fibras musculares (grau de edema tecidual), presença de infiltrado neutrofílico (resposta inflamatória) e destruição do sarcolema (necrose celular).

Com a finalidade de permitir a comparação entre os grupos, estabeleceu-se um sistema de graduação dos parâmetros (escores) observados (Tabela 1): para cada parâmetro, foi calculada a média aritmética dos valores encontrados nos 20 campos contados em cada lâmina, correspondendo a um animal de cada grupo.

O processamento imuno-histoquímico foi realizado com utilização do anticorpo primário cleaved anticaspase-3, anti-rato $\left(\mathrm{DAKO}{ }^{\circledR} \mathrm{A} / \mathrm{S}\right.$, Denmark 492), revelador DAB (3,3-diaminobenzidina) Sigma Co-USAD5632 e obtenção de expressão caspase-3 na coloração marrom. 
Tabela 1 - Parâmetros de avaliação da lesão celular e graduação da intensidade das lesões encontradas em campos analisados pelo patologista na microscopia óptica, pela coloração em hematoxilina e eosina

\begin{tabular}{lcccc}
\hline & \multicolumn{3}{c}{ Escore } & \\
\cline { 2 - 5 } & Grau 0 & Grau 1 & Grau 2 & Grau 3 \\
& Ausente & Leve & Moderado & Acentuado \\
\hline $\begin{array}{l}\text { Dissociação de fibras } \\
\text { musculares (edema) }\end{array}$ & Ausente & $\begin{array}{c}\text { 1 espaço claro } \\
\text { por campo (100x) }\end{array}$ & $\begin{array}{c}\text { 2-4 espaços claros } \\
\text { por campo (100x) }\end{array}$ & $>$ por campo (100x) \\
Infiltrado neutrofílico & & 1-10 neutrófilos & $11-20$ neutrófilos & $>$ 20 neutrófilos \\
& Ausente & por campo (400x) & por campo (400x) & por campo (400x) \\
Necrose de fibras musculares & Ausente & 1 fibra & 2-3 fibras & $>4$ fibras \\
& & por campo (100x) & por campo (100x) & por campo (100x) \\
\hline
\end{tabular}

A quantificação da imuno-histoquímica foi realizada por captura de 10 imagens por lâmina de cada animal, utilizando-se o microscópio óptico marca Nikon $^{\circledR}$, em conexão com microcomputador Pentium ${ }^{\circledR}$ III, $650 \mathrm{MHz}, 256 \mathrm{MB}$ de Ram, HD de 40 GB e sistema operacional Microsoft Windows ${ }^{\circledR} 98$ SE.

A quantificação da densidade de cor marrom foi efetuada utilizando-se o filtro RGB, cor de fundo azul, em intervalo de cor de zero a 147, que corresponde ao espectro de cor marrom ${ }^{18-20}$. A análise foi realizada com uso de programa informatizado de análise de imagens (ImageLAB ${ }^{\circledR} 2.3$ ), baseado em princípios de espectrofotometria ${ }^{19}$. Para a quantificação de cada parâmetro, foram captados 10 campos microscópicos, com locais demarcados (tamanho padronizado em $3 \mathrm{~cm}$ ) das áreas perinuclear e perivascular da fibra muscular, representativas de apoptose dos núcleos celulares de interesse, em cada imagem.

Para cada lâmina, correspondendo a um animal de cada grupo, foi calculada a média aritmética dos valores de cada área (perivascular e perinuclear), nos 10 campos contados em relação à área não-corada pela imunohistoquímica, e expressa em porcentagem (Figura 3).

O estudo estatístico foi realizado pelos testes de Kruskal-Wallis e Mann-Whitney para comparar os subgrupos entre si. Considerou-se estatisticamente significantes valores de $\mathrm{p}<0,05$, com intervalo de confiança de $95 \%$.

\section{Resultados}

\section{Análise morfológica pela microscopia óptica}

A dissociação de fibras musculares, o infiltrado neutrofílico intersticial e a necrose de fibras musculares foram os parâmetros analisados pela microscopia óptica. Apesar de ter sido investigada, a variável necrose não foi encontrada nesse modelo experimental. Após análise morfológica, foram calculadas as médias do escore estabelecido para cada variável analisada em todos os subgrupos.

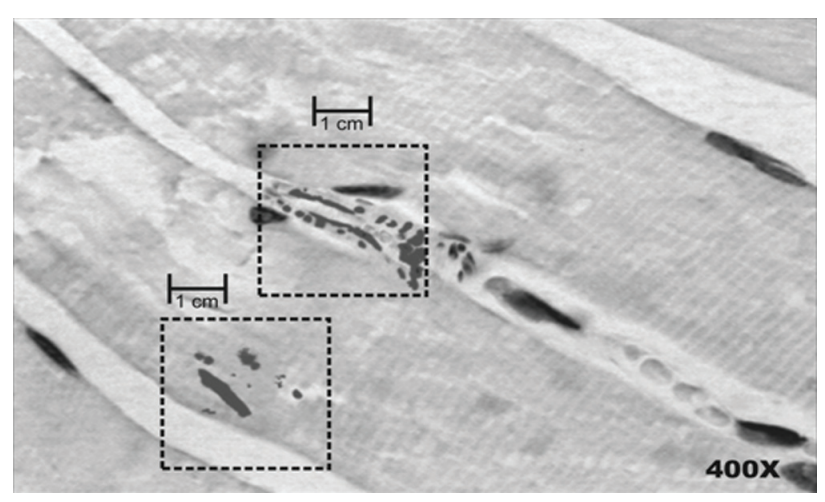

Figura 3 - Fotomicrografia das áreas perivascular e perinuclear da fibra muscular, selecionadas (marcadas) com coloração azul expressando a caspase-3 (imunocoloração 400x)

Não foram encontradas alterações desses parâmetros no GS e, em razão disso, o grupo não pôde ser comparado aos dos subgrupos experimentais.

Os resultados dos cálculos das médias e os respectivos desvios padrão dos escores nos diversos subgrupos estão demonstrados nas Tabelas 2 e 3 e nas Figuras 4 e 5. 


\section{Descrição morfológica da dissociação de fibras}

$\mathrm{O}$ afastamento das fibras musculares pode ser interpretado como sendo devido à interposição de líquido intersticial e, portanto, uma expressão de edema e intumescimento do tecido muscular.

O músculo dos animais do GS, quer aqueles que ficaram 4 ou 24 horas em observação, não mostrou afastamento das fibras musculares, que apresentavam sua característica disposição fusiforme e com alinhamento uniforme em todo o campo examinado.

Os animais do grupo A3 e alguns animais do grupo B3 foram os que apresentaram os escores mais favoráveis, com predominância do grau leve de afastamento, caracterizado pela presença de um espaçamento por campo e o restante das fibras fusiformes uniformemente alinhadas.

Os animais dos grupos A1 e A2 e alguns do grupo B3 apresentaram os escores mais representativos da dissociação de fibras de grau moderado, onde dois a três espaçamentos foram identificados em cada campo, e o restante das fibras manteve a característica fusiforme, porém já apresentava certo desalinhamento com tortuosidade. Os animais dos grupos B1 e B2 apresentaram grau acentuado de dissociação de fibras, caracterizado por numerosos espaços entre as fibras que ficaram tortuosas e desalinhadas.

\section{Descrição morfológica do infiltrado neutrofilico}

A presença de células inflamatórias no interstício foi caracterizada como um indicador de intensidade de resposta celular inflamatória do tecido muscular à isquemia e reperfusão.

O músculo dos animais do GS, quer aqueles que ficaram 4 ou 24 horas em observação, não mostrou infiltrado leucocitário entre as fibras musculares, que apresentavam sua característica disposição fusiforme e com alinhamento uniforme em todo o campo examinado.

Os animais do grupo A3 e alguns do grupo B3 foram os que apresentaram os escores mais favoráveis, com

Tabela 2 - Médias do escore da análise da dissociação de fibras para os subgrupos A1 (sem PTX), A2 (com PTX-R) e A3 (com PTX-I/R), submetidos a isquemia de $6 \mathrm{~h}$ + reperfusão de 4 h, e B1 (sem PTX), B2 (com PTX-R) e B3 (com PTX-I/R), submetidos a isquemia de $6 \mathrm{~h}+$ reperfusão de $24 \mathrm{~h}$

\begin{tabular}{lcccccc}
\hline & A1 & A2 & A3 & B1 & B2 & B3 \\
\hline R1 & 1,8 & 1,7 & 0,8 & 2,3 & 2,3 & 1,5 \\
R2 & 1,8 & 1,7 & 0,9 & 2,3 & 2 & 1,2 \\
R3 & 1,8 & 1,7 & 0,5 & 2 & 2 & 0,7 \\
R4 & 1,7 & 1,8 & 0,9 & 2,2 & 2,3 & 1,1 \\
R5 & 1,7 & 1,8 & 0,7 & 2 & 2,3 & 0,9 \\
R6 & 1,7 & 1,7 & 0,8 & 2,3 & 2 & 1,1 \\
R7 & 1,7 & 1,8 & 0,5 & 2 & 2,3 & 1,2 \\
R8 & 1,7 & 1,7 & 0,9 & 2,3 & 2,3 & 0,9 \\
R9 & 1,7 & 1,8 & 0,7 & 2 & 2,2 & 1,1 \\
R10 & 1,7 & 1,7 & 0,9 & 2,2 & 2,3 & 0,8 \\
Média & 1,73 & 1,74 & 0,76 & 2,16 & 2,20 & 1,05 \\
Desvio padrão & 0,05 & 0,05 & 0,16 & 0,14 & 0,14 & 0,23 \\
\hline
\end{tabular}

PTX = pentoxifilina; PTX-I/R = pentoxifilina na isquemia e na reperfusão; PTX-R = pentoxifilina na reperfusão. 


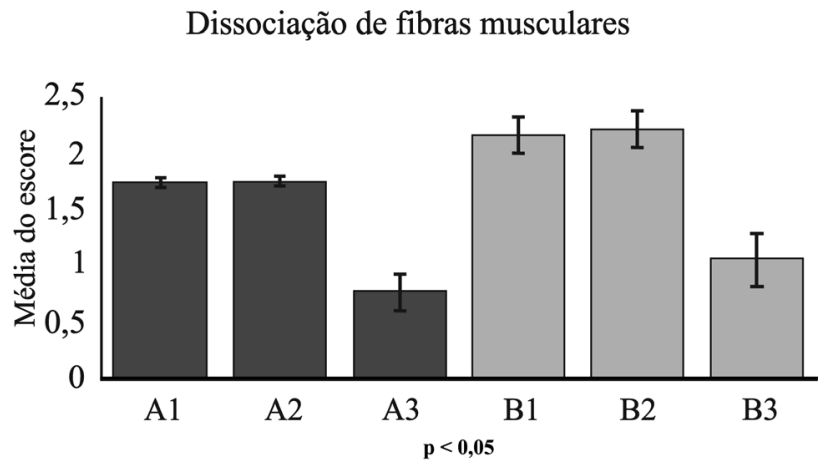

Figura 4 - Médias do escore da análise da dissociação de fibras para os subgrupos A1 (sem PTX), A2 (com PTX-R) e A3 (com PTX-I/R), submetidos a isquemia de $6 h$ + reperfusão de 4 h, e B1 (sem PTX), B2 (com PTX-R) e B3 (com PTX-I/R), submetidos a isquemia de $6 \mathrm{~h}+$ reperfusão de $24 \mathrm{~h}$

PTX = pentoxifilina; PTX-I/R = pentoxifilina na isquemia e na reperfusão; PTX-R = pentoxifilina na reperfusão.

predominância do grau leve de infiltrado neutrofílico, caracterizado pela presença de uma até 10 células por campo, geralmente em posição próxima aos vasos presentes nos espaços intercelulares.
Os animais dos grupos A1 e A2 e alguns do grupo B3 foram os que apresentaram os escores mais representativos da infiltração neutrofílica de grau moderado, onde de 10 a 20 células inflamatórias se infiltraram no espaço intercelular e em locais mais distantes do espaço vascular.

Os animais dos grupos B1 e B2 apresentaram grau acentuado de infiltração de neutrófilos, caracterizado por numerosas células, mais de 20 por campo, entre as fibras musculares.

\section{Resultados da imuno-histoquímica}

A imunocoloração foi avaliada pela expressão da caspase-3 ativada nas áreas perivascular e perinuclear da fibra muscular. Foi padronizada uma área de um quadrado de $3 \times 3 \mathrm{~cm}$ para marcar a região escolhida para expressão da caspase-3 em percentual de imunocoloração das respectivas áreas, pelo programa computadorizado de imageLab ${ }^{\circledR}$.

Tabela 3 - Médias do escore da análise do infiltrado neutrofílico para os subgrupos A1 (sem PTX), A2 (com PTX-R) e A3 (com PTX-I/R), submetidos a isquemia de $6 \mathrm{~h}$ + reperfusão de 4 h, e B1 (sem PTX), B2 (com PTX-R) e B3 (com PTX-I/R), submetidos a isquemia de $6 \mathrm{~h}+$ reperfusão de $24 \mathrm{~h}$

\begin{tabular}{lcccccc}
\hline & A1 & A2 & A3 & B1 & B2 & B3 \\
\hline R1 & 1,2 & 1,5 & 1 & 2,1 & 2 & 1,5 \\
R2 & 1,8 & 2 & 1 & 2 & 1,9 & 1,6 \\
R3 & 2,1 & 1,7 & 0,8 & 2,1 & 2,1 & 1 \\
R4 & 2,2 & 2,1 & 1 & 2,1 & 2,6 & 1,5 \\
R5 & 1,7 & 1,8 & 0,8 & 1,9 & 2,1 & 1 \\
R6 & 1,7 & 2 & 1 & 2,2 & 2,1 & 1,3 \\
R7 & 1,7 & 1,2 & 0,8 & 2,1 & 2 & 1,6 \\
R8 & 2 & 1,7 & 1 & 2 & 2 & 1 \\
R9 & 2 & 2,1 & 0,8 & 1,9 & 2,1 & 1,5 \\
R10 & 2 & 2 & 1 & 2,1 & 2 & 1 \\
Média & 1,84 & 1,81 & 0,92 & 2,05 & 2,09 & 1,3 \\
Desvio padrão & 0,29 & 0,29 & 0,10 & 0,10 & 0,19 & 0,27 \\
\hline PTX - pra
\end{tabular}

PTX = pentoxifilina; PTX-I/R = pentoxifilina na isquemia e na reperfusão; PTX-R = pentoxifilina na reperfusão. 


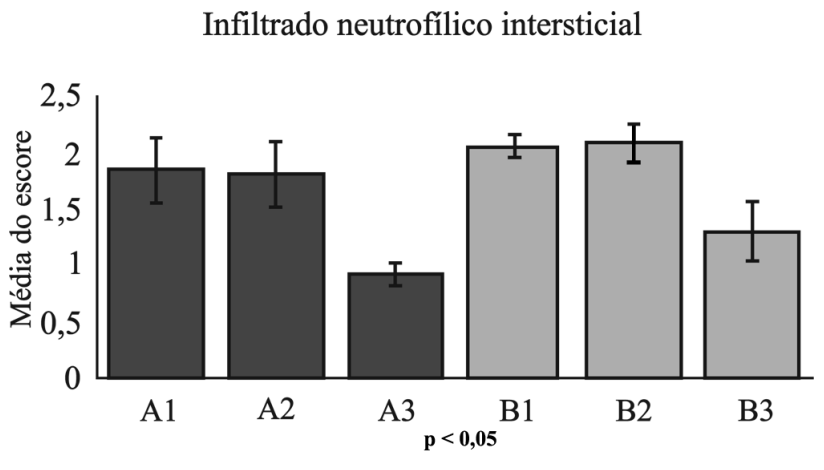

Figura 5 - Médias do escore da análise do infiltrado neutrofílico para os subgrupos A1 (sem PTX), A2 (com PTX-R) e A3 (com PTX-I/R), submetidos a isquemia de $6 h$ + reperfusão de 4 h, e B1 (sem PTX), B2 (com PTX-R) e B3 (com PTX-I/R), submetidos a isquemia de $6 \mathrm{~h}+$ reperfusão de $24 \mathrm{~h}$

PTX = pentoxifilina; PTX-I/R = pentoxifilina na isquemia e na reperfusão; PTX-R = pentoxifilina na reperfusão.

Os resultados das médias, em percentual, da expressão da caspase-3 de todos os grupos estão demonstrados nas Tabelas 4, 5 e 6 e nas Figuras 6, 7 e 8.
O emprego da pentoxifilina avaliada na área perinuclear no período de reperfusão, quer na fase precoce $(\mathrm{A} 2=0,39 \pm 0,11)$ ou na fase tardia $(\mathrm{B} 2=0,47 \pm 0,21)$, não mostrou diferenças; contudo, em ambos os casos a expressão foi significantemente menor nos animais que não fizeram uso da droga. Quando avaliada a sua aplicação em ambos os períodos (isquemia e reperfusão), na fase precoce ( $\mathrm{A} 3=0,27 \pm 0,12$ ) ou tardia $(\mathrm{B} 3=0,37 \pm 0,15)$, ambos os casos apresentaram resultados significantemente inferiores aos animais que não receberam a droga, embora não tenha havido diferenças no momento de aplicação da droga. Desse modo, a pentoxifilina foi igualmente favorável na manifestação da apoptose nos neutrófilos perinucleares tanto na fase precoce como na tardia, mostrando uma ação imediata e sustentada.

A comparação da expressão da caspase entre as áreas perinucleares e perivasculares, nos diferentes períodos de aplicação e fases da avaliação (Figura 8),

Tabela 4 - Médias em percentual da expressão da caspase-3 na área perivascular para os subgrupos A1 (sem PTX), A2 (com PTX-R) e A3 (com PTX-I/R), submetidos a isquemia de $6 \mathrm{~h}+$ reperfusão de $4 \mathrm{~h}$, e B1 (sem PTX), B2 (com PTX-R) e B3 (com PTX-I/R), submetidos a isquemia de $6 \mathrm{~h}+$ reperfusão de $24 \mathrm{~h}$

\begin{tabular}{lccccccc}
\hline & $\mathbf{S}$ & $\mathbf{A 1}$ & $\mathbf{A 2}$ & $\mathbf{A 3}$ & $\mathbf{B 1}$ & $\mathbf{B 2}$ & $\mathbf{B 3}$ \\
\hline R1 & 1,02 & 3,57 & 1,44 & 0,76 & 3,63 & 2,93 & 0,75 \\
R2 & 0,84 & 3,61 & 2,69 & 0,56 & 3,63 & 1,84 & 1,04 \\
R3 & 0,82 & 3,13 & 2,06 & 0,72 & 4,31 & 2,96 & 1,03 \\
R4 & 0,96 & 2,70 & 2,52 & 0,58 & 4,03 & 2,57 & 1,02 \\
R5 & 0,92 & 3,31 & 2,04 & 0,68 & 4,11 & 1,86 & 1,44 \\
R6 & 0,80 & 2,28 & 2,07 & 0,91 & 3,53 & 1,87 & 1,23 \\
R7 & & 3,10 & 1,95 & 0,55 & 4,75 & 1,75 & 1,26 \\
R8 & & 2,71 & 1,44 & 0,49 & 5,17 & 2,02 & 1,53 \\
R9 & & 2,14 & 1,85 & 0,57 & 5,176 & 1,88 & 0,96 \\
R10 & 2,49 & 2,25 & 0,89 & 3,06 & 2,45 & 0,77 \\
Média & 0,89 & 2,90 & 2,03 & 0,67 & 4,30 & 2,21 & 1,10 \\
Desvio padrão & 0,079 & 0,52 & 0,40 & 0,15 & 0,79 & 0,47 & 0,26 \\
Coeficiente de variação & $8,88 \%$ & $17,86 \%$ & $19,84 \%$ & $21,86 \%$ & $18,50 \%$ & $21,30 \%$ & $23,58 \%$ \\
\hline
\end{tabular}

PTX = pentoxifilina; PTX-I/R = pentoxifilina na isquemia e na reperfusão; PTX-R = pentoxifilina na reperfusão. 
mostra que a pentoxifilina foi significantemente favorável para diminuir a apoptose em ambas as áreas e com maior intensidade quando aplicada nos dois períodos (isquemia e reperfusão).

\section{Discussão}

Diante de um quadro isquêmico dos membros inferiores, a prioridade é restabelecer o fluxo sangüíneo. A extensão e gravidade das alterações morfológicas e funcionais teciduais dependem fundamentalmente do período de oclusão do fluxo sangüíneo arterial ${ }^{1-6}$.

Trabalhos em animais de experimentação têm procurado estudar modelos que possam reproduzir as condições de isquemia encontradas em seres humanos e, com isso, ter um melhor entendimento da fisiopatologia da doença e das alternativas terapêuticas ${ }^{2,21,22}$.

Optou-se pelo modelo de obstrução isolada da artéria ilíaca comum por atender aos objetivos de tentar simular a ocorrência de isquemia resultante de obstru-

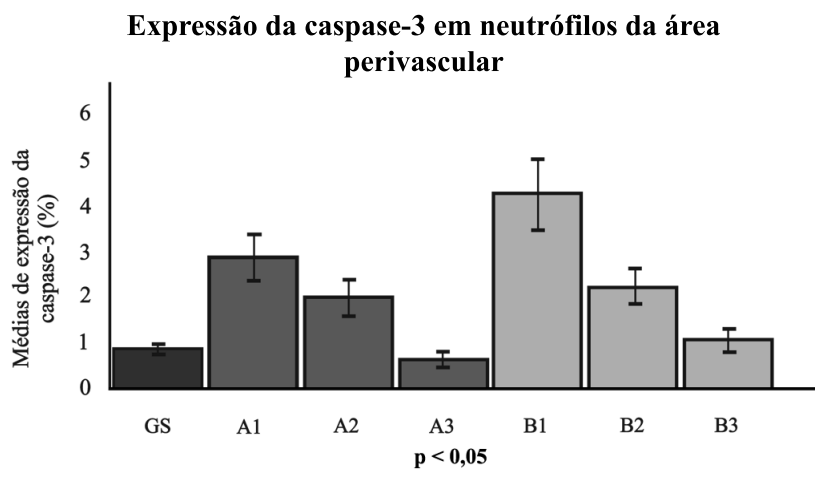

Figura 6 - Médias, em percentual, da expressão da caspase-3 na área perivascular para os subgrupos A1 (sem PTX), A2 (com PTX-R) e A3 (com PTX-I/R), submetidos a isquemia de $6 \mathrm{~h}+$ reperfusão de $4 \mathrm{~h}$, e B1 (sem PTX), B2 (com PTX-R) e B3 (com PTX$\mathrm{I} / \mathrm{R})$, submetidos a isquemia de $6 \mathrm{~h}+$ reperfusão de $24 \mathrm{~h}$

PTX = pentoxifilina; $\mathrm{PTX}-\mathrm{I} / \mathrm{R}=$ pentoxifilina na isquemia e na reperfusão; PTX-R = pentoxifilina na reperfusão.

ções arteriais agudas, que ocorrem com freqüência na prática médica ${ }^{8}$. Os testes do projeto-piloto mostraram

Tabela 5 - Médias, em percentual, da expressão da caspase-3 na área perinuclear para os subgrupos A1 (sem PTX), A2 (com PTX-R) e A3 (com PTX-I/R), submetidos a isquemia de $6 \mathrm{~h}+$ reperfusão de $4 \mathrm{~h}$, e B1 (sem PTX), B2 (com PTX-R) e B3 (com PTX-I/R), submetidos a isquemia de $6 \mathrm{~h}+$ reperfusão de $24 \mathrm{~h}$

\begin{tabular}{lccccccc}
\hline & $\mathbf{S}$ & $\mathbf{A 1}$ & $\mathbf{A 2}$ & $\mathbf{A 3}$ & $\mathbf{B 1}$ & $\mathbf{B 2}$ & $\mathbf{B 3}$ \\
\hline R1 & 0,07 & 1,13 & 0,18 & 0,28 & 0,99 & 0,63 & 0,19 \\
R2 & 0,03 & 1,38 & 0,65 & 0,51 & 0,83 & 0,34 & 0,30 \\
R3 & 0,07 & 1,05 & 0,37 & 0,36 & 0,49 & 0,35 & 0,24 \\
R4 & 0,1 & 1,32 & 0,35 & 0,19 & 0,38 & 0,26 & 0,38 \\
R5 & 0,13 & 1,33 & 0,33 & 0,21 & 0,73 & 0,65 & 0,15 \\
R6 & 0,12 & 1,19 & 0,38 & 0,21 & 0,91 & 0,39 & 0,38 \\
R7 & & 0,90 & 0,42 & 0,27 & 1,10 & 0,24 & 0,50 \\
R8 & & 1,13 & 0,40 & 0,06 & 1,53 & 0,72 & 0,51 \\
R9 & & 0,23 & 0,44 & 0,40 & 1,12 & 0,84 & 0,65 \\
R10 & & 1,05 & 0,40 & 0,22 & 0,97 & 0,27 & 0,43 \\
Média & 0,09 & 1,07 & 0,39 & 0,27 & 0,91 & 0,47 & 0,37 \\
Desvio padrão & 0,0372 & 0,3305 & 0,1159 & 0,1260 & 0,3284 & 0,2191 & 0,1568 \\
Coeficiente de variação & $42,97 \%$ & $30,86 \%$ & $29,57 \%$ & $46,50 \%$ & $36,28 \%$ & $46,72 \%$ & $42,03 \%$ \\
\hline
\end{tabular}

PTX = pentoxifilina; PTX-I/R = pentoxifilina na isquemia e na reperfusão; PTX-R = pentoxifilina na reperfusão. 
Expressão da caspase-3 em neutrófilos da área perinuclear

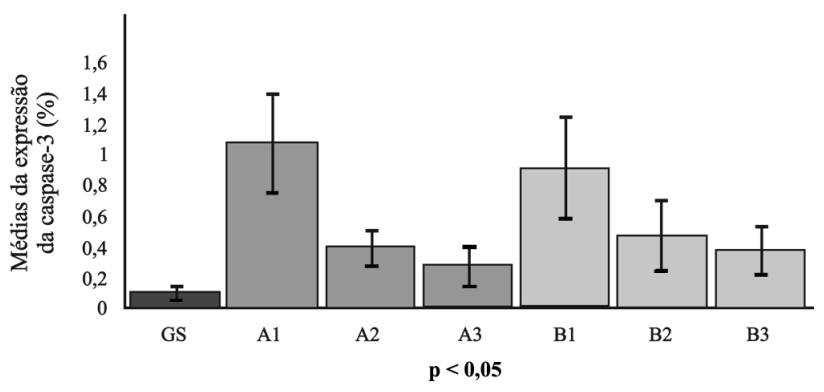

Figura 7 - Médias, em percentual, da expressão da caspase-3 na área perinuclear para os subgrupos A1 (sem PTX), A2 (com PTX-R) e A3 (com PTX-I/R), submetidos a isquemia de $6 \mathrm{~h}+$ reperfusão de $4 \mathrm{~h}$, e B1 (sem PTX), B2 (com PTX-R) e B3 (com PTX$\mathrm{I} / \mathrm{R})$, submetidos a isquemia de $6 \mathrm{~h}+$ reperfusão de $24 \mathrm{~h}$

PTX = pentoxifilina; PTX-I/R = pentoxifilina na isquemia e na reperfusão; PTX-R = pentoxifilina na reperfusão.

que o edema e a cianose apresentados pelo membro pélvico do animal após o clampeamento arterial foram parâmetros confiáveis para comprovar a isquemia desse membro.

Há consenso na literatura em relação ao músculo sóleo como sendo o mais adequado para o estudo das lesões decorrentes da isquemia e reperfusão ${ }^{4,7,23,24}$, uma vez que o tipo de fibra e a localização dos grupamentos musculares estão relacionados ao grau de lesão causado pelo fenômeno de isquemia e reperfusão. O músculo sóleo, por possuir $91 \%$ de fibras tipo I, torna-se mais sensível aos processos de isquemia. Sua maior sensibilidade ao estresse oxidativo, resultante da produção de espécies reativas de oxigênio, é explicada pela sua via metabólica para obtenção de energia, preferencialmente a oxidação de glicerídeos e, em menor grau, a via oxidativa glicogênica ${ }^{4,7}$.

O músculo esquelético é considerado como sendo resistente às lesões isquêmicas, apesar da sensibilidade específica do músculo sóleo ${ }^{4,7,23,24}$. A literatura revela que o músculo esquelético suporta bem a isquemia por períodos de até 4 horas, apesar de apresentar algumas repercussões locais e sistêmicas.

As lesões morfológicas começam a se manifestar cerca de 15 minutos após a isquemia (reperfusão) e aumentam progressivamente à medida que aumenta a resposta inflamatória. Ficam mais evidentes com 4 horas e atingem o pico máximo após 24 horas $^{21,25,26}$. Desse modo, procurou-se estudar alterações tidas como precoces (4 horas) e tardias (24 horas). Períodos anteri-

Tabela 6 - Comparações das médias da expressão da caspase-3 entre as áreas perinuclear e perivascular nos subgrupos A1 (sem PTX), A2 (com PTX-R) e A3 (com PTX-I/R), submetidos a isquemia de $6 \mathrm{~h}+$ reperfusão de 4 h, e B1 (sem PTX), B2 (com PTX-R) e B3 (com PTX-I/R), submetidos a isquemia de $6 \mathrm{~h}+$ reperfusão de $24 \mathrm{~h}$

\begin{tabular}{|c|c|c|c|c|c|}
\hline \multicolumn{3}{|c|}{ Área perinuclear } & \multicolumn{3}{|c|}{ Área perivascular } \\
\hline & Média & Desvio padrão & & Média & Desvio padrão \\
\hline GS & 0,09 & 0,0372 & GS & 0,45 & 0,04 \\
\hline GA1 & 1,07 & 0,3305 & GA1 & 2,9 & 0,52 \\
\hline GA2 & 0,39 & 0,1159 & GA2 & 2,03 & 0,4 \\
\hline GA3 & 0,27 & 0,126 & GA3 & 0,67 & 0,15 \\
\hline GB1 & 0,91 & 0,3284 & GB1 & 4,3 & 0,79 \\
\hline GB2 & 0,47 & 0,2191 & GB2 & 2,21 & 0,47 \\
\hline GB3 & 0,37 & 0,1568 & GB3 & 1,1 & 0,26 \\
\hline
\end{tabular}

GS = grupo simulado; GA = grupo A; GB = grupo B; PTX = pentoxifilina; PTX-I/R = pentoxifilina na isquemia e na reperfusão; PTX-R = pentoxifilina na reperfusão. 
ores a 4 horas trazem resultados irrelevantes, e períodos posteriores a 24 horas mostram que há uma tendência à diminuição das lesões ${ }^{25}$.

\section{Expressão da caspase-3 nas áreas perinuclear e perivascular}

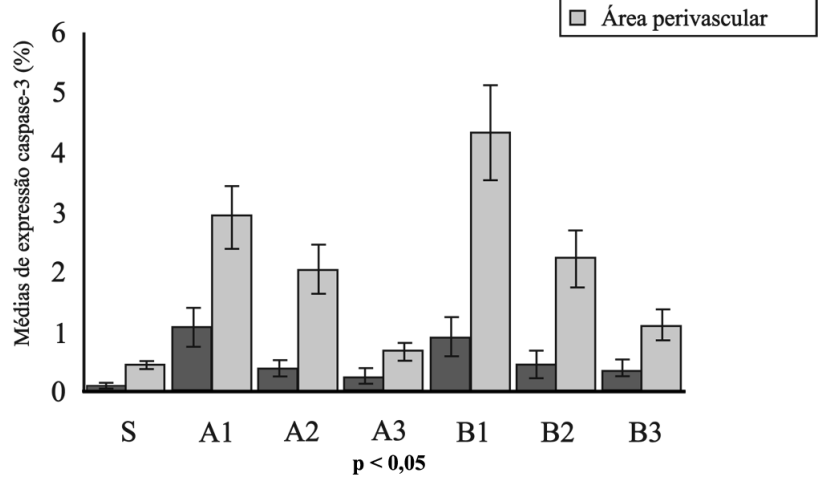

Figura 8 - Comparações das médias da expressão da caspase-3 entre a área perinuclear e perivascular nos subgrupos A1 (sem PTX), A2 (com PTX-R) e A3 (com PTX-I/R), submetidos a isquemia de $6 \mathrm{~h}$ + reperfusão de 4 h, e B1 (sem PTX), B2 (com PTX-R) e B3 (com PTX-I/R), submetidos a isquemia de $6 \mathrm{~h}+$ reperfusão de $24 \mathrm{~h}$

PTX = pentoxifilina; PTX-I/R = pentoxifilina na isquemia e na reperfusão; PTX-R = pentoxifilina na reperfusão.

Um dos primeiros sinais que ocorre após a desobstrução arterial é representado pelo edema intersticial. O extravasamento de líquidos intravascular para o interstício é ocasionado por alterações biofísicas (mecânicas), pelo aumento da pressão intracapilar, e posteriormente por alterações bioquímicas, pelo aumento da permeabilidade capilar, que resultam em acúmulo de líquidos entre as fibras musculares, levando à dissociação dessas fibras. Quanto maior o edema, maior a dissociação das fibras, havendo uma relação temporal: quanto maior o período de isquemia, maior o edema $a^{6,27,28}$. A infiltração de líquido intersticial afasta as fibras musculares, e o exame de campos representativos em mesmo aumento microscópico pôde caracterizar o grau dessa infiltração.

O processo isquêmico desencadeia uma esperada reação inflamatória, caracterizada pela diapedese de leucócitos que se interpõem, a partir dos espaços vasculares, entre a fibras musculares. Em princípio, a concentração leucocitária se faz ao redor dos vasos e, com o avançar do processo, espalha-se pelo interstício das fibras musculares. Os neutrófilos são os mais freqüentemente encontrados até 24 horas. Representam uma resposta do organismo aos processos humorais desencadeados pelo processo inflamatório, visando ao isolamento do fator agressor e início do processo de reparação da lesão,6,27,24,29,30. Além do edema e da infiltração leucocitária e em conseqüência da ausência de oxigênio ou substrato oxidável, progressivamente as células musculares podem entrar em necrose ${ }^{24}$.

A expressão da caspase-3 pode ser utilizada como um marcador de apoptose. Efetuadores pertencentes à família das proteases, as caspases são proteases cisteínas ativadas por fatores liberados pela mitocôndria no processo de desencadeamento de morte celular e podem ser identificadas por processo imunohistoquímico ${ }^{3,18,27}$.

A marcação em fibras musculares (perinucleares) teve a intenção de identificar a apoptose pela expressão da caspase-3 especificamente sobre o tecido muscular esquelético. A marcação dos neutrófilos na área perivascular foi a tentativa de expressar a magnitude da reação inflamatória. Quanto maior o sofrimento leucocitário, expresso pela apoptose em neutrófilos, mais grave é a lesão originada da isquemia e reperfusão $0^{3,18,27}$.

Tem-se buscado formas de abolir ou minimizar os efeitos das espécies reativas de oxigênio ou radicais livres ${ }^{5,21,23,24,31,32}$. Para isso, tem-se utilizado substâncias inibidoras da formação dos radicais livres, conhecidas como antioxidantes (vitamina E, ácido ascórbico, deferoxamina, estreptoquinase, alopurinol, sais de magnésio, aminoesteróides, quelantes do ferro e antiinflamatórios não-esteróides), substâncias varredoras dessas substâncias (scavengers) $)^{31}$ e processos de précondicionamento isquêmico, onde supressões parciais e progressivas de oxigenação antecedem uma supressão sustentada mais prolongada ${ }^{5,31}$. Cada uma dessas proposições têm mostrado vantagens e desvantagens na dependência dos modelos de isquemia e reperfusão, nas doses das substâncias utilizadas, nos períodos em que são empregadas, nos parâmetros que são usados para avaliação dos resultados, entre outras variáveis ${ }^{6}$. 
A pentoxifilina tem ação antioxidante, inibindo diretamente o ânion superóxido e, de forma indireta, bloqueando a ação da xantina oxidase. Além disso, parece diminuir a resposta inflamatória, pela redução da migração de neutrófilos e outros mecanismos, reduzindo a liberação de citocinas, fator de necrose tumoral, fator ativador de plaquetas e liberação de endotelina, que é uma potente substância vasoconstrictora ${ }^{9,10,17}$. Considerando esses suportes teóricos, propôs-se a utilização dessa droga para testar sua atuação em isquemia e reperfusão de músculo esquelético.

A análise e interpretação dos resultados histológicos, no tocante aos parâmetros estudados, mostraram algumas variações significativas que mereceram atenção.

O GS mostrou que o trauma anestésico e operatório não trouxe influências significativas sobre o tecido muscular e pode ser utilizado como ponto de comparação com os outros dois grupos de estudo. Consideraram-se os achados obtidos como padrão de normalidade.

Foi significativa a ausência de alterações morfológicas típicas da necrose tecidual. As fibras musculares nos animais submetidos a 6 horas de isquemia não mostraram reações necróticas evidenciáveis 4 e 24 horas após o restabelecimento da perfusão tecidual. O fato pode demonstrar a resistência do tecido muscular à agressão isquêmica, mas esta deve estar associada também à capacidade ou peculiaridade da circulação arterial colateral dos membros posteriores dos ratos e ao nãocomprometimento do retorno venoso.

Alguns autores referidos na literatura, que usaram o mesmo tempo de clampeamento arterial em ratos, também não lograram identificar áreas de necrose de fibras musculares, embora tenham observado a ocorrência de outras alterações morfológicas e aumento da creatina fosfoquinase, parâmetro indicado de necrose ${ }^{2,21}$.

A dissociação de fibras, que expressa a presença de edema intersticial nos animais observados após 4 horas de reperfusão, mostrou que a aplicação da pentoxifilina no período de reperfusão não foi diferente daquela dos animais do grupo-controle. Porém, quando a droga foi administrada em dois períodos (isquemia e reperfusão), houve uma significativa diminuição da expressão do edema. O perfil repetiu-se na observação de 24 horas, onde o grupo-controle foi semelhante ao grupo de aplicação da droga na reperfusão e significantemente menor no grupo de aplicação nos dois períodos. Desse modo, pode-se concluir que a pentoxifilina teve ação sobre o processo, melhorando a expressão do edema. Como o componente inicial de perda de líquido intravascular é mecânico (biofísico), pelo aumento da pressão intracapilar, e posteriormente bioquímico, envolvendo a vitalidade celular e atividades enzimáticas que levam ao aumento da permeabilidade vascular e relação de forças de tensão osmótica dos tecidos, pode-se inferir que a capacidade hemorreológica da pentoxifilina deva ter interferência favorável, impedindo ou dificultando a perda intravascular de líquidos.

A relação do edema tecidual é tempo-dependente. Com 4 horas de reperfusão, o edema é menos importante do que com 24 horas. Nas fases precoce e tardia, a pentoxifilina mostrou-se eficaz na redução do edema. Pode-se imaginar que, por ter reduzido o edema já na fase inicial, isso representaria um efeito conseqüente quando da observação da fase tardia, porém, como a metabolização da droga é lenta, pode-se inferir que ainda haja ação efetiva da droga também no período tardio.

Trabalhos relatam a relação do aumento progressivo das alterações morfológicas quando se observa um período de até 24 horas após isquemia em músculo esquelético $^{25,26}$. Depois desse período, ocorre tendência à normalização dos tecidos quanto maior for o tempo de observação após a isquemia ${ }^{25}$.

A mesma relação teórica sobre o edema pode se estabelecer para a avaliação da migração de neutrófilos. Sabe-se que a pentoxifilina tem capacidade de inibir a migração dos leucócitos ${ }^{9,14,15,17}$. Como a migração leucocitária é um processo ativo, que depende da liberação local de substâncias ativas, como citocinas, fator de necrose tumoral, fator ativador de plaquetas e endotelina, e esses fatores humorais são modulados 
pela droga, é lícito inferir que a sua ação foi favorável também nesse aspecto sobre a lesão de isquemia e reperfusão muscular.

Os resultados da pesquisa são concordantes com outros da literatura biomédica. Estudo sobre o clampeamento da aorta abdominal de ratos por 90 minutos e reperfusão por 60 minutos mostrou que pentoxifilina atenuou o edema e o infiltrado leucocitário da musculatura do membro pélvico dos animais, quando utilizada nos períodos de isquemia e reperfusão ${ }^{12}$. Em outro modelo de isquemia de músculo esquelético por 5 horas e observação pós-isquemia por 20 horas, conseguiu-se estabelecer que, quanto maior for a dose da pentoxifilina, melhores são os efeitos protetores pela redução de edema, infiltrado leucocitário e áreas de necrose das fibras musculares, assim como pela redução do fator ativador de plaquetas ${ }^{11}$. Em outro modelo experimental de isquemia de membros pélvicos de ratos pelo clampeamento da aorta abdominal, observou-se que a repercussão local e pulmonar no grupo que se utilizou pentoxifilina apresentou redução dos níveis de fator de necrose tumoral alfa (TNF- $\alpha$ ) e infiltrado leucocitário no músculo e pulmão, quando comparado ao grupocontrole e ao da dexametasona ${ }^{33}$.

A proposição de analisar a expressão de caspase-3 nas áreas perivascular e perinuclear da fibra muscular foi baseada em trabalhos de literatura que encontraram maior evidência de apoptose somente em infiltrado neutrofílico e vasos de músculos submetidos a estresse oxidativo, demonstrando que a fibra muscular é mais resistente à apoptose e mais sensível à necrose ${ }^{27,34,35}$.

A avaliação da apoptose pela expressão imunohistoquímica da caspase-3 mostrou que ela é pouco importante no tecido muscular normal, representado pelos animais do GS. A expressão em neutrófilos em área perinuclear se mostrou muito discreta quando comparada à da área perivascular, e ambas foram muito inferiores aos valores dos animais submetidos a isquemia e reperfusão. Portanto, a expressão da apoptose em neutrófilos nas condições do GS, onde houve apenas a influência do trauma anestésico e operatório, é praticamente inexistente.
No entanto, a expressão nos animais submetidos a isquemia e reperfusão na área perivascular, na fase precoce ou tardia, é significantemente maior, assim como na área perinuclear, tanto na fase precoce como na tardia. A expressão foi tempo-dependente na área perivascular, onde, na fase precoce, o índice foi menor que na fase tardia. $\mathrm{Na}$ área perinuclear, os índices foram equivalentes, quer na fase precoce, quer na fase tardia. Os achados permitem inferir que os neutrófilos perinucleares e núcleos da fibra muscular são menos susceptíveis de entrarem em processo de apoptose que os neutrófilos perivasculares, revelando que os últimos podem estar mais diretamente envolvidos nos processos de lesão tecidual ou mais expostos aos fatores agressores da lesão de isquemia e reperfusão. $\mathrm{O}$ fator agressivo principal de ativação da cascata das caspases é atribuído à liberação de espécies reativas de oxigênio durante a fase de reperfusão ${ }^{18,27,34}$.

A pentoxifilina mostrou eficácia na diminuição da expressão da caspase-3, que é um monitor da apoptose celular, quando houve a somatória das doses, aplicadas nos dois períodos.

A pentoxifilina, nesse modelo experimental, mostrou-se eficaz tanto na atenuação da reação inflamatória pela diminuição do edema e do infiltrado leucocitário como na diminuição da expressão da caspase-3, o que a coloca de acordo com as manifestações da literatura sobre os seus mecanismos de ação sobre o fenômeno de isquemia e reperfusão.

Os achados desta pesquisa permitem concluir que a pentoxifilina é uma droga que deve continuar fazendo parte do arsenal de fármacos que vêm sendo investigados para abolir ou atenuar as lesões de isquemia e reperfusão e que o modelo ora proposto é viável e acrescenta um novo parâmetro de avaliação das lesões de isquemia e reperfusão, pela expressão da caspase-3 em infiltrado leucocitário do tecido muscular.

\section{Conclusões}

O modelo de isquemia e reperfusão em músculo sóleo de ratos produziu alterações morfológicas, tempodependentes do período de reperfusão, porém, sem evidências de necrose ou apoptose muscular. 
A pentoxifilina mostrou-se favorável na atenuação das alterações morfológicas em tecido muscular, pela redução do edema e do infiltrado neutrofílico entre as fibras musculares, quando aplicada no início da isquemia e da reperfusão.

Mostrou-se também favorável na atenuação da expressão da caspase-3, no infiltrado leucocitário nas áreas perivascular e perinuclear, sendo mais significante quando aplicada no início da isquemia e da reperfusão.

\section{Referências}

1. Silva JCCB, Burihan E. Diagnóstico clínico da isquemia crítica dos membros. Rev Bras Clin Ter. 1999;25:71-9.

2. Francisco Neto A, Silva JCCB, Fagundes DJ, et al. Estudo das alterações oxidativas, da capacidade antioxidante total e do óxido nítrico, em ratos submetidos à isquemia e reperfusão de membros posteriores. Acta Cir Bras. 2005;20:134-9.

3. Lockshin RA, Zakeri Z. Caspase-independent cell deaths. Curr Opin Cell Biol. 2002; 14:727-33.

4. Poli Figueiredo LF, Cruz Jr. RJ, Morsch RD. Desvascularização e reperfusão no trauma vascular. In: Murilo XX. Trauma vascular. $1^{\mathrm{a}}$ ed. Rio de Janeiro: Revinter; 2005. p. 628-33.

5. Bitu-Moreno J, Gregório EA, Maffei FHA. Effect of alpha-tocopherol on the ischemia/reperfusion lesions induced in the hindlimb of rats. Acta Cir Bras. 2001;16:68-74.

6. Silveira M, Yoshida WB. Isquemia e reperfusão em músculo esquelético: mecanismos de lesão e perspectivas de tratamento. J Vasc Bras. 2004;3:367-78.

7. Badhwar A, Dungey AA, Harris KA, et al. Limitations of ischemic tolerance in oxidative skeletal muscle: perfusion vs tissue protection. J Surg Res. 2003;109:62-7.

8. Quiñones-Baldrich WJ, Chervu A, Hernandez JJ, Colburn M, Moore WS. Skeletal muscle function after ischemia: "no reflow" versus reperfusion injury. J Surg Res.1991;51:5-12.

9. Zhang M, Xu YJ, Saini HK, Turan B, Liu PP, Dhalla NS. Pentoxifylline attenuates cardiac dysfunction and reduces TNF-alpha level in ischemic-reperfused heart. Am J Physiol Heart Circ Physiol. 2005;289:H832-9.

10. Uguralp S, Bay Karabulut A, Mizrak B. Effects of pentoxifylline and vitamin $\mathrm{E}$ on the bilateral ovary after experimental ovarian ischemia. Eur J Pediatr Surg. 2005;15:107-13.

11. Adams JG Jr., Dhar A, Shukla SD, Silver D. Effect of pentoxifylline on tissue injury and platelet-activating factor production during ischemia-reperfusion injury. J Vasc Surg. 1995;21:742-8; discussion 748-9.

12. Kishi M, Tanaka H, Seiyama A, et al. Pentoxifylline attenuates reperfusion injury in skeletal muscle after partial ischemia. Am J Physiol. 1998;274(5 Pt 2):H1435-42.

13. Yildirim S, Tok H, Koksal H, Erdem L, Baykan A. Allopurinol plus pentoxifylline in hepatic ischaemia/ reperfusion injury. Asian J Surg. 2002;25:149-53.
14. Clark SC, Rao JN, Flecknell PA, Dark JH. Pentoxifylline is as effective as leukocyte depletion for modulating pulmonary reperfusion injury. J Thorac Cardiovasc Surg. 2003;126:2052-7.

15. Dominguez-Jimenez C, Sancho D, Nieto M, et al. Effect of pentoxifylline on polarization and migration of leukocytes. $\mathrm{J}$ Leukoc Biol. 2002;71:588-96.

16. Sener G, Akgun U, Satiroglu H, Topaloglu U, Keyer-Uysal M. The effect of pentoxifylline on intestinal ischemia/reperfusion injury. Fundam Clin Pharmacol. 2001;15:19-22.

17. Schratzberger P, Dunzendorfer S, Reinisch N, et al. Mediator-dependent effects of pentoxifylline on endothelium for transmigration of neutrophils. Immunopharmacology. 1999;41:65-75.

18. Gown AM, Willingham MC. Improved detection of apoptotic cells in archival paraffin sections: Immunohistochemistry using antibodies to cleaved caspase 3 . J Histochem Cytochem. 2002;50:449-54.

19. Borra RC, Pizarro PJ. Imagelab98. Disponível em: http//www.brasil.terravista.pt/PraiaBrava/1563/imgelab.html. Acessado: 04/04/2005.

20. Kohlberger PD, Breitenecker F, Kaider A, et al. Modified true-color computer-assisted image analysis versus subjective scoring of estrogen receptor expression in breast cancer: a comparison. Anticancer Res. 1999;19:2189-93.

21. Souza-Moraes MR, David-Filho R, Baptista-Silva JC, et al. Effect of antibodies to intercellular adhesion molecule type 1 on the protection of distant organs during reperfusion syndrome in rats. Braz J Med Biol Res. 2003;36:605-12.

22. Fagundes DJ, Taha MO. Modelo animal de doença: critérios de escolha e espécies de animais de uso corrente. Acta Cir Bras. 2004;19:59-65.

23. Francischetti I, Maffei FHA, Bitu-Moreno J, et al. Ação do ácido trissódio-cálcio-dietileno-triaminopentaacético (CaNa3DTPA) nas lesões de isquemia-reperfusão em membro posterior de rato. Acta Cir Bras. 2002;17:1-17.

24. Torres JMS, Guimarães SB, Vasconcelos PRL, Martins MCR, Chaves CR, Vasconcelos PRC. Efeitos metabólicos da 1-alanil-glutamina em ratos submetidos à isquemia da pata traseira esquerda seguida de reperfusão. Acta Cir Bras. 2003;18:39-44.

25. Wiersema AM, Oyen WJ, Dirksen R, Verhofstad AA, Corstens FH, van der Vliet JA. Early assessment of skeletal muscle damage after ischaemia-reperfusion injury using Tc-99m-glucarate. Cardiovasc Surg. 2000;8:186-91.

26. Lazarus B, Messina A, Barker JE, et al. The role of mast cells in ischaemia-reperfusion injury in murine skeletal muscle. $\mathrm{J}$ Pathol. 2000;191:443-8.

27. Cowled PA, Leonardos L, Millard SH, Fitridge RA. Apoptotic cell death makes a minor contribution to reperfusion injury in skeletal muscle in the rat. Eur $\mathrm{J}$ Vasc Endovasc Surg. 2001;21:28-34.

28. Souza AC, Piccinato CE, Cherri J, Moriya T. Mecanismos celulares e moleculares da lesão de isquemia e reperfusão de músculo esquelético. Cir Vasc Angiol. 2001;17:80-6.

29. Piccinato CE, De Domenico Jr. A, Jordäo Jr. AA, Vannucchi $\mathrm{H}$. Skeletal muscle ischemia and reperfusion in rats increase lipid peroxidation in rats. Acta Cir Bras. 2004;19:578-81. 
30. Alves WF, Guimaräes SB, Vasconcelos PRC, Vasconcelos PRL. Repercussões da L-alanil-glutamina sobre as concentrações de lactato e lactato desidrogenase (LDH) em pacientes com isquemia crítica dos membros inferiores submetidos à revascularização distal. Acta Cir Bras. 2003;18:209-15.

31. Bitu-Moreno J, Francischetti I, Hafner L. Lesöes de isquemia-reperfusão em músculos esqueléticos: fisiopatologia e novas tendências de tratamento, com ênfase em reperfusão controlada. J Vasc Bras. 2002;1:113-20.

32. David Filho R, Moraes MRS, Silva JCCB, et al. Blockage of intracellular adhesion molecule-1 (ICAM-1) in the prevention of reperfusion lesion in the skeletal musculature of EPM-1 Wistar rats. Acta Cir Bras. 2004;19:590-6.

33. Berkan $\mathrm{O}$, Gol MK, Icagasioglu $\mathrm{S}$, et al. Sialic acid is a marker of lung injury following lower extremities ischemia/reperfusion. Eur $\mathrm{J}$ Vasc Endovasc Surg. 2004; 27:553-8.
34. Knight KR, Messina A, Hurley JV, Zhang B, Morrison WA, Stewart AG. Muscle cells become necrotic rather than apoptotic during reperfusion of ischaemic skeletal muscle. Int J Exp Pathol.1999;80:169-75.

35. Leeuwenburgh C, Gurley CM, Strotman BA, Dupont-Versteegden EE. Age-related differences in apoptosis with disuse atrophy in soleus muscle. Am J Physiol Regul Integr Comp Physiol. 2005;288:R1288-96.

Correspondência:

José Lacerda Brasileiro

Rua Gonçalo Alves, 59, Vivendas do Bosque

CEP 79021-182 - Campo Grande, MS

Tel.: (67) 9281.8940, (67) 3326.2842

E-mail: jlbrasileiro@brturbo.com.br 